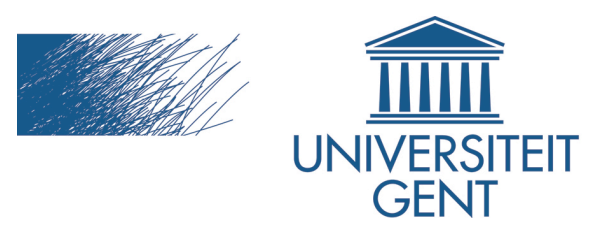

\title{
biblio.ugent.be
}

The UGent Institutional Repository is the electronic archiving and dissemination platform for all UGent research publications. Ghent University has implemented a mandate stipulating that all academic publications of UGent researchers should be deposited and archived in this repository. Except for items where current copyright restrictions apply, these papers are available in Open Access.

This item is the archived peer-reviewed author-version of:

Can the prevalence of high blood drug concentrations in a population be estimated by analysing oral fluid? A study of tetrahydrocannabinol and amphetamine Hallvard Gjerde $^{a}$ and Alain Verstraete ${ }^{b}$

Forensic Sci Int. 2010 Feb 25;195(1-3):153-9

To refer to or to cite this work, please use the citation to the published version:

Gjerde H, Verstraete A. Can the prevalence of high blood drug concentrations in a population be estimated by analysing oral fluid? A study of tetrahydrocannabinol and amphetamine.

Forensic Sci Int. 2010 Feb 25;195(1-3):153-910.1016/j.forsciint.2009.12.011i 


\section{Can the prevalence of high blood drug concentrations in a population be estimated by analysing oral fluid? A study of tetrahydrocannabinol and amphetamine.}

Hallvard Gjerde $^{\mathrm{a}}$ and Alain Verstraete ${ }^{\mathrm{b}}$

${ }^{a}$ Norwegian Institute of Public Health, Division of Forensic Toxicology and Drug Abuse,

P.O. Box 4404 Nydalen, 0403 Oslo, Norway

${ }^{\mathrm{b}}$ Ghent University, Faculty of Medicine and Health Sciences and Ghent University Hospital, Laboratory of Clinical Biology, De Pintelaan 185, 9000 Ghent, Belgium

Corresponding author:

Dr. Hallvard Gjerde

Norwegian Institute of Public Health

Division of Forensic Toxicology and Drug Abuse

P.O. Box 4404 Nydalen

NO-0403 Oslo

Norway

Telephone: +4721077953

Fax: +4721077878

Email: Hallvard.Gjerde@,fhi.no 


\section{Can the prevalence of high blood drug concentrations in a population be estimated by analysing oral fluid? A study of tetrahydrocannabinol and amphetamine.}

\section{A B S T R A C T}

Aim: To study several methods for estimating the prevalence of high blood concentrations of tetrahydrocannabinol and amphetamine in a population of drug users by analysing oral fluid (saliva).

Methods: Five methods were compared, including simple calculation procedures dividing the drug concentrations in oral fluid by average or median oral fluid/blood (OF/B) drug concentration ratios or regression coefficients and more complex Monte Carlo simulations.

Populations of 311 cannabis users and 197 amphetamine users from the Rosita-2 Project were studied.

Results: The results of a feasibility study suggested that the Monte Carlo simulations might give better accuracies than simple calculations if good data on $\mathrm{OF} / \mathrm{B}$ ratios is available. If using only 20 randomly selected OF/B ratios, a Monte Carlo simulation gave the best accuracy but not the best precision. Dividing by the $\mathrm{OF} / \mathrm{B}$ regression coefficient gave acceptable accuracy and precision, and was therefore the best method. None of the methods gave acceptable accuracy if the prevalence of high blood drug concentrations was less than $15 \%$.

Conclusion: Dividing the drug concentration in oral fluid by the $\mathrm{OF} / \mathrm{B}$ regression coefficient gave an acceptable estimation of high blood drug concentrations in a population, and may therefore give valuable additional information on possible drug impairment, e.g. in roadside surveys of drugs and driving. If good data on the distribution of $\mathrm{OF} / \mathrm{B}$ ratios are available, a Monte Carlo simulation may give better accuracy.

Key-words: amphetamine; tetrahydrocannabinol; blood; oral fluid; prevalence; population; Monte Carlo simulation, linear regression 


\section{Introduction}

Blood and urine are the most commonly used biological fluids for drug analysis. Urine sample analysis is used for the detection of drug use, while serum, plasma or whole blood analysis is required in cases of therapeutic drug monitoring, cases of suspected over-dosing, and when assessing possible drug impairment, e.g. among suspected drugged drivers. Oral fluid (mixed saliva) may also be analysed to detect and monitor drug use, and the use for this purpose is increasing [1-4]. Oral fluid is an easily available medium that can be collected with noninvasive methods without the intrusion of privacy. Oral fluid has about the same detection window (time range) as blood regarding alcohol and drugs [5]. A fairly small amount of oral fluid is needed for the analyses of alcohol and drugs [6], and the oral fluid sample may be collected within 2-5 minutes by using a simple, commercially available collection device [7].

Alcohol and most drugs enter oral fluid from blood by a passive diffusion process dependent on the compound's physicochemical properties, primarily $\mathrm{pKa}$, protein binding, lipophilicity, molecular weight and spatial configuration [5]. Significant inter- and intra-subject variability in oral fluid/whole blood (OF/B) ratios has been observed [8-9], and the wide range of OF/B ratios does not allow reliable calculation of the drug concentrations in blood from drug concentrations in oral fluid. There are, however, positive correlations between drug concentrations in oral fluid and blood, varying from one drug to another [9-12]. For alcohol, the concentration in oral fluid reflects very well the blood alcohol concentration $[1,4]$.

The technique used for sampling oral fluid may affect the analytical result. Physical or chemical stimulation of the production of oral fluid is often used in order to increase the sample volume; however, the concentration of drugs might in these cases be different from concentrations in non-stimulated oral fluid [8, 13-15]. The sampling device itself may also affect the analytical results because the recoveries of some drugs might vary from one device to another [7-8]. When comparing data from different studies on drug concentrations in oral fluid, it is therefore important to bear in mind the effect of different sampling procedures.

Oral fluid is often being collected in epidemiological studies of the prevalence of drug use, e.g. in roadside surveys of drug use among drivers. Our hypothesis is that the distribution of drug concentrations in oral fluid in a population of drug users is related to the distribution of drug concentrations in blood, and that the distribution of concentrations in oral fluid may be 
used to estimate the prevalence of blood drug concentrations above certain concentration limits. Such a method for utilising drug concentrations in oral fluid may give useful additional information in epidemiological studies.

A correlation between drug concentrations in oral fluid and blood was found for amphetamine, tetrahydrocannabinol (THC), and other drugs in the Rosita-2 study $[9,16]$. We examined the distribution of concentrations of amphetamine and THC from that study using the EasyFit software (www.mathwave.com) and found that two two-parameter probability distributions fitted the distributions of concentrations of both drugs in both blood and oral fluid well: the Weibull and Lognormal distributions. We also found that these probability distributions also fitted concentration data for zopiclone, diazepam, THC and codeine in samples of oral fluid from a study of random drivers [17]. We propose that if the oral fluid drug concentration distribution in a population fits a distribution model, a similar, plausible distribution in whole blood may be estimated. A number of representative OF/B ratios are needed for this estimation; to obtain $\mathrm{OF} / \mathrm{B}$ ratios samples of oral fluid and blood must be obtained and analysed in the same way as for the study population.

The aim of the present study was to evaluate several methods for estimating the prevalence of high drug concentrations by calculating the accuracy (bias; deviation between estimated and observed prevalence of drug concentrations above chosen limits in blood) and precision (relative standard deviation, RSD) of the methods. Concentration data for THC and amphetamine observed in the Rosita-2 Project $[9,16]$ were used to compare the estimation procedures. To our knowledge, Rosita- 2 is the only survey where a sufficiently large number of drug concentration data for blood and oral fluid is available.

\section{Materials and methods}

\subsection{Study population}

The Rosita-2 Project $[9,16]$ included drivers who were apprehended by police in several countries suspected for driving under the influence of drugs. Samples of oral fluid were collected by using the Intercept Oral Specimen Collection Device (OraSure Technologies, Bethlehem PA, USA), and whole blood samples were also obtained. The samples were analysed for a number of psychoactive drugs. All drivers who tested positive for THC or amphetamine in samples of oral fluid, and from whom results for analysis of THC and 
amphetamine in blood were available, were selected for this study. Analytical findings have been presented elsewhere $[9,16]$.

\subsection{Analytical method}

Samples of whole blood and oral fluid were analysed by chromatographic-mass spectrometric methods. The drug concentrations in undiluted oral fluid were in some countries calculated by using the average dilution factor, in other countries by determining the dilution for each single sample by weighing the samples. References to different methods have been presented elsewhere [9].

\subsection{Simple calculation methods for estimation of blood drug concentrations}

A correlation between drug concentrations in oral fluid and blood has been found for both amphetamine and THC; for THC this correlation was more evident when examining the logarithmic values [9]. Therefore some simple methods were used to calculate virtual drug concentrations in blood based on actual concentrations in oral fluid, assuming that the distribution of the virtual blood drug concentrations would match the actual distribution of drug concentrations in blood.

\section{Method A: Divide concentration in OF by average}

The average $\mathrm{OF} / \mathrm{B}$ ratio was calculated, and each drug concentration in oral fluid was divided by the average ratio giving a set of virtual blood drug concentrations. The prevalence of blood drug concentrations above or equal to a chosen concentration limit (e.g. THC $\geq 6.0 \mathrm{ng} / \mathrm{ml}$ ) was estimated as the percentage of virtual blood drug concentrations above or equal to same limit. For the feasibility study all OF/B ratios were used to calculate the average, while for the validation studies 20 randomly selected $\mathrm{OF} / \mathrm{B}$ ratios were used.

\section{Method B: Divide concentration in OF by regression coefficient}

The linear regression coefficient (slope) between oral fluid and blood was calculated using the "least squares" method by employing the LINEST function in Microsoft Excel with intercept at zero. Each drug concentration in oral fluid was divided by the regression coefficient giving a set of virtual blood drug concentrations that were used for calculations as described above. 


\section{Method C: Divide concentration in OF by median}

The median $\mathrm{OF} / \mathrm{B}$ ratio was calculated and used to calculate the prevalence and each drug concentration in oral fluid was divided by the median $\mathrm{OF} / \mathrm{B}$ ratio giving a set of virtual blood drug concentrations that were used for calculations as described above.

\subsection{Monte Carlo simulations}

A Monte Carlo simulation is a method evaluating a deterministic model using sets of random numbers (or rather, pseudorandom numbers) as inputs, often iteratively [18-19]. Sets of random numbers complying with a chosen probability distribution function are generated, these numbers are included in more or less complex computations, and the outcome is evaluated mathematically.

If the distribution of blood drug concentrations in a population of drug users can be defined by a mathematical probability function, we expect that the distribution of drug concentrations in oral fluid can also be defined by a similar mathematical probability function, except that the parameters describing the distributions are different. The difference in the parameters are defined by the $\mathrm{OF} / \mathrm{B}$ drug concentration ratios of that particular population.

If the blood drug concentration and $\mathrm{OF} / \mathrm{B}$ ratio of each single individual in a population is known, the drug concentrations in oral fluid may be calculated by simply multiplying each blood drug concentration with the $\mathrm{OF} / \mathrm{B}$ ratio. The distribution of drug concentrations in oral fluid may then be determined accurately. If the individual OF/B ratios are not known, the distribution of drug concentrations in oral fluid may be approximated by multiplying each single blood drug concentration with a random $\mathrm{OF} / \mathrm{B}$ ratio from an equivalent population (later called "representative OF/B ratios" in this report). This approximation is used in the Monte Carlo simulation described below. This procedure is expected to give the best approximation if the $\mathrm{OF} / \mathrm{B}$ ratio is independent of blood drug concentrations.

If we know the drug concentrations in oral fluid, and the individual OF/B ratios are not known, we cannot simply estimate the blood drug concentration by dividing the concentration in oral fluid by a random $\mathrm{OF} / \mathrm{B}$ ratio; this procedure would generate an extremely wide distribution of virtual blood drug concentrations that would be very much different from the actual distribution. Therefore, a Monte Carlo simulation may be used to solve this problem. In 
our method, we generated a random distribution of simulated blood drug concentrations complying with a chosen probability distribution function (Lognormal or Weibull), then multiplied each simulated blood concentration with a random $\mathrm{OF} / \mathrm{B}$ ratio to obtain simulated oral fluid drug concentrations. If the distribution of the simulated oral fluid drug concentration matched the actual distribution observed in the population being studied, the simulated blood drug concentration distribution was expected to describe a plausible distribution of blood drug concentrations. To obtain a match between the simulated and actual drug concentration distributions, the parameters describing the simulated blood drug concentration distribution were changed in an iterative process until a match was obtained.

The distributions that fitted the observed data for oral fluid and blood best were the Weibull and Lognormal distributions. It is easy to make calculations for the Lognormal probability distribution using a Microsoft Excel spreadsheet, whereas for the Weibull distributions it is more difficult without using so-called ad-ins for Excel. We have therefore described the use of a spreadsheet performing Lognormal calculations below, and a modification using the Weibull probability distribution for drug concentrations in blood.

\section{Method D: Monte Carlo simulation using Lognormal probability distribution}

In the first step of our procedure, we calculated the actual lognormal parameters meanlog $\left(\mathrm{M}_{\mathrm{OF}}\right)$ and $s d \log \left(\mathrm{S}_{\mathrm{OF}}\right)$ for the observed distribution of drug concentrations in oral fluid: the meanlog is the mean of the natural logarithmic values of drug concentrations in oral fluid, and $s d l o g$ is the standard deviation of the logarithmic values.

An iterated Monte Carlo simulation was performed to determine a lognormal blood drug concentration distribution that would fit the observed oral fluid drug concentration. The simulation procedure is presented in Figure 1. Using a Microsoft Excel spreadsheet, 5000 random numbers (representing simulated blood drug concentrations) fitting a lognormal distribution with meanlog $\mathrm{M}_{\mathrm{Blood}}$ and $s d \log \mathrm{S}_{\mathrm{Blood}}$ were generated by using a combination of the inverse logarithmic function and the random function: LOGINV(RAND(),meanlog,sdlog) (use a semicolon instead of a comma as separator if your spreadsheet uses a decimal comma). As initial values, 1.00 was chosen for both $\mathrm{M}_{\mathrm{Blood}}$ and $\mathrm{S}_{\mathrm{Blood}}$. Each of the 5000 random numbers was multiplied with one of the 20 selected OF/B ratios giving a simulated oral fluid drug concentration. The Lognormal distribution parameters meanlog $\mathrm{M}_{\mathrm{OFS}}$ and $s d \log \mathrm{S}_{\mathrm{OFS}}$ of the 5000 simulated oral fluid drug concentrations were calculated and compared with the 
meanlog $\mathrm{M}_{\mathrm{OF}}$ and $s d \log \mathrm{S}_{\mathrm{OF}}$ for the actual drug concentration distribution. In each of the following iteration steps of the procedure, the values for meanlog and sdlog for the blood concentration distribution $\left(\mathrm{M}_{\mathrm{Blood}}\right.$ and $\left.\mathrm{S}_{\mathrm{Blood}}\right)$ were slightly increased or decreased to obtain a converging fit between simulated and observed values for meanlog and $s d l o g$. When the meanlog and sdlog for the simulated oral fluid drug concentrations ( $\mathrm{M}_{\mathrm{OFS}}$ and $\mathrm{S}_{\mathrm{OFS}}$ ) matched the actually observed meanlog and sdlog for the study population $\left(\mathrm{M}_{\mathrm{OF}}\right.$ and $\left.\mathrm{S}_{\mathrm{OF}}\right)$, with a maximum difference in meanlog and sdlog values of $<0.01\left(\left|\mathrm{M}_{\mathrm{OFS}}-\mathrm{M}_{\mathrm{OF}}\right|<0.01\right.$ and $\mid \mathrm{S}_{\mathrm{OFS}}-$ $\left.\mathrm{S}_{\mathrm{OF}} \mid<0.01\right)$, the input values for blood meanlog and blood sdlog $\left(\mathrm{M}_{\mathrm{Blood}}\right.$ and $\left.\mathrm{S}_{\mathrm{Blood}}\right)$ used for the simulation were describing a plausible blood drug concentration distribution of the study population. The spreadsheet formulae and instructions for use are shown in Table 1.

$<$ Insert Table 1 approximately here $>$

$<$ Insert Figure 1 approximately here $>$

\section{Method E: Monte Carlo simulation using the Weibull probability distribution}

A Microsoft Excel spreadsheet can be used to generate random distributions fitting Weibull distributions, but cannot easily be used to calculate the distribution parameters scale and shape of an actual population. Since the Weibull and Lognormal distributions are similar, we used Lognormal calculations to compare the simulated and actual distributions of drug concentrations in oral fluid as a approximation, but generated blood drug concentration distributions complying with the Weibull distribution using the following formula in Microsoft Excel: $\mathrm{c}^{*}(-\mathrm{LN}(1-\operatorname{RAND}()))^{\wedge}(1 / \mathrm{m})$, where $\mathrm{c}=$ scale and $\mathrm{m}=$ shape. This formula was used in cells B7 to B5006 in the spreadsheet presented in Table 1, and scale and shape replaced meanlog and sdlog in cells A3 and A4.

\subsection{Feasibility study}

An initial feasibility study was performed using the total population of subjects positive for amphetamine or THC in oral fluid, not sub-populations. For the simple calculation methods, the average and median of all $\mathrm{OF} / \mathrm{B}$ ratios and the regression coefficient for the total number of samples were used. For the Monte Carlo simulations, $20 \mathrm{OF} / \mathrm{B}$ ratios were systematically selected to represent the total distribution of $\mathrm{OF} / \mathrm{B}$ ratios. For amphetamine, $20 \mathrm{OF} / \mathrm{B}$ ratios 
5.00 to the 98.00 percentile (i.e. percentiles $5.00,9.89,14.79, \ldots, 93.11,98.00$ ). To exclude the most extreme ratios for $\mathrm{THC}, 20 \mathrm{OF} / \mathrm{B}$ ratios were selected evenly from the 8.00 to the 94.00 percentile.

\subsection{Validation studies: determination of accuracy and precision}

In most study situations, a limited number of representative $\mathrm{OF} / \mathrm{B}$ ratios will be available for the actual oral fluid sampling method used and population studied. Therefore, a realistic procedure should be based on a reasonable number of OF/B ratios; we have used 20 ratios.

Twenty subjects with drug detected both in oral fluid and blood were selected at random from each of the populations of THC-positive and amphetamine-positive individuals by using the random function in a Microsoft Excel spreadsheet, and the OF/B drug concentration ratios were calculated. This selection of $\mathrm{OF} / \mathrm{B}$ ratios was repeated five times, thus generating six sets of representative $\mathrm{OF} / \mathrm{B}$ ratios for each drug in order to determine the precision of this method. The remaining subjects were used as study populations. The accuracy was calculated as average percent of the six sub-populations studied, while the precision was calculated as the relative standard deviation of the accuracies.

\section{Results and Discussion}

Tetrahydrocannabinol (THC) was detected in 311 samples of oral fluid from the Rosita-2 Project, and was thus the most commonly detected drug. Of the 311 persons who had THC concentrations above the analytical cut-off in oral fluid, 277 had also THC concentrations above the analytical cut-off in blood. Thus, altogether $277 \mathrm{OF} / \mathrm{B}$ ratios for THC were available. The $\mathrm{OF} / \mathrm{B}$ ratios ranged from 0.006 to 569 with an average of 34.1 and a median value of 15.4 , the apparent SD was 63.4. The distribution was thus very much skewed. The 10 and 90 percentiles corresponded to 0.1 and 5 times the median, respectively.

Amphetamine was the second most prevalent drug found in the Rosita-2 project. Altogether 197 subjects provided oral fluid samples that were positive for amphetamine, 187 of these subjects were also positive for amphetamine in blood. The OF/B ratios ranged from 0.27 to 210 with an average of 19 and a median of 12 , the apparent SD was 26 . The distribution was 
very much skewed, and the 10 and 90 percentiles corresponded to 0.2 and 3 times the median, respectively.

\subsection{Feasibility study of the estimation methods}

The prevalences of blood drug concentrations equal to or above specified limits determined in the feasibility study are presented in Tables 2 and 3.

$<$ Insert Table 2 approximately here $>$

$<$ Insert Table 3 approximately here $>$

When dividing the drug concentrations by the average OF/B ratio the obtained results matched very well the actual blood concentration data as far as the prevalence of blood amphetamine concentrations $\geq 800$ and $\geq 1000 \mathrm{ng} / \mathrm{ml}$ was concerned, however, for THC the accuracy was poor, mainly due to the fact that the large number of very high $\mathrm{OF} / \mathrm{B}$ ratios observed for THC gave a high average $\mathrm{OF} / \mathrm{B}$ ratio. If using the average $\mathrm{OF} / \mathrm{B}$ ratio for estimating the THC concentration distribution in blood, the highest $\mathrm{OF} / \mathrm{B}$ ratios must therefore be excluded.

Dividing by the regression coefficient gave over-estimation of high drug concentrations for THC, but to a less extent for amphetamine. In total, using the regression coefficient seemed to give slightly more accurate estimations. Dividing by the median OF/B values gave poor accuracy for both THC and amphetamine and seems to be the least feasible method.

As far as Monte Carlo simulations were concerned, the Weibull simulation gave a better estimation of the actual drug concentration distributions in blood compared to the Lognormal simulation, but the results for the Lognormal simulation were also quite acceptable. The results suggest that good estimates may be obtained at optimal conditions, which may be 
obtained if large populations are studied, the Lognormal or Weibull distribution functions fit the drug concentration data in oral fluid, and a large number of representative $\mathrm{OF} / \mathrm{B}$ ratios are available.

The Lognormal simulation gave a slight over-estimation of high drug concentrations in blood, while the Weibull simulation gave a slight under-estimation, probably because of the differences in the shapes of the Lognormal and Weibull probability distributions: a fitting Lognormal distribution has a larger right tail than a Weibull distribution, see Figure 2.

\subsection{Validation studies using 20 random OF/B ratios}

In most study situations, a limited number of representative $\mathrm{OF} / \mathrm{B}$ ratios will be available for the actual oral fluid sampling method used and population studied. Therefore, a realistic procedure for the estimation of blood drug concentrations in a population should be based on a reasonable number of $\mathrm{OF} / \mathrm{B}$ ratios; we decided to use 20 ratios for this study.

We chose $20 \mathrm{OF} / \mathrm{B}$ ratios at random from each population or drug users. In order to use independent data, the individuals providing the $\mathrm{OF} / \mathrm{B}$ ratios that were used for the calculations were removed from the study population. We found that a random selection of $20 \mathrm{OF} / \mathrm{B}$ ratios in some cases contained several extremely high or extremely low values $(<0.1$ times or $>5$ times the median value), and thus disabled the Monte Carlo simulation procedures (because the distribution of $\mathrm{OF} / \mathrm{B}$ ratios was too large compared to the distribution of concentrations observed in oral fluid samples of the studied population). To eliminate this cause of error, we excluded OF/B ratios below 0.1 times the median and above 5 times the median of the selected $\mathrm{OF} / \mathrm{B}$ ratios as outliers, and selected $\mathrm{OF} / \mathrm{B}$ ratios until 20 ratios complying with this requirement were obtained. The validation results for THC and amphetamine for the Rosita-2 population are presented in Tables 4-5.

\footnotetext{
$<$ Insert Table 4 approximately here $>$ $<$ Insert Table 5 approximately here $>$
} 
The precision and accuracy were not as good as for the initial feasibility study, primarily because that the estimations were based on a random selection of only $20 \mathrm{OF} / \mathrm{B}$ ratios which have large variation. The RSD for the average $\mathrm{OF} / \mathrm{B}$ ratios in the six selections were $14.0 \%$ and $32.3 \%$ for THC and amphetamine, respectively. The precision and accuracy for the estimation of blood amphetamine concentrations were worse than for THC, mainly because of larger variation in the $\mathrm{OF} / \mathrm{B}$ ratios used for the calculations, but also because the population was smaller.

The accuracies for all estimation procedures were unacceptable for estimating the prevalence of THC concentrations $\geq 10.0 \mathrm{ng} / \mathrm{ml}$ (actual prevalence of $14.5 \%$ ) and amphetamine concentrations $\geq 1000 \mathrm{ng} / \mathrm{ml}$ (actual prevalence of $11.9 \%$ ). The precision RSD of all estimation procedures were greater than $25 \%$ for most estimations of blood amphetamine concentrations, but less than or equal to $25 \%$ for all estimation procedures when measuring THC.

For the simple calculation methods, best accuracy and precision was obtained when dividing the drug concentrations in oral fluid by the regression coefficient. For the Monte Carlo simulations, the Weibull simulation gave somewhat better accuracy but worse precision than the Lognormal simulation. In total, dividing by the regression coefficient seemed to be the best method for the populations of THC and amphetamine users in this study when the calculations were based on only $20 \mathrm{OF} / \mathrm{B}$ ratios. Better results are expected if a larger number of $\mathrm{OF} / \mathrm{B}$ data are included in the calculations. We also expect that the methods would give better precision for drugs with more narrow distribution of OF/B ratios than THC and amphetamine.

\section{Conclusion}

The results suggest that the prevalence of blood drug concentrations above chosen limits may be roughly estimated by using analytical results for oral fluid when dividing drug concentrations in oral fluid by the $\mathrm{OF} / \mathrm{B}$ regression coefficient or by using Monte Carlo simulations. Dividing by the regression coefficient gave better results than more advanced Monte Carlo simulations when only 20 OF/B ratios were available for the calculations. However, the results of the feasibility study suggested that Monte Carlo simulations may give better accuracy if $\mathrm{OF} / \mathrm{B}$ ratios that are representative for the distribution in a larger population are available. 


\section{Acknowledgements}

The collection and analysis of blood and oral fluid samples were financed by the European Commission through the Rosita-2 Project. The study design, calculations and preparation of this report were partly funded by the European Commission through the DRUID Project (Driving Under the Influence of Drugs, Alcohol and Medicines), which is within the EU 6th Framework Programme for Research and Technological Development. The funding sources had no role in the interpretation of data, in the writing of the report, or in the decision to submit the paper for publication, and are not liable for any use that may be made of the information contained therein. This report reflects only the authors' view.

Thanks to the Rosita-2 partners who collected and analysed the samples of blood and oral fluid: National Institute of Criminalistics and Criminology (Brussels, Belgium), National Public Health Institute (Helsinki, Finland), Saarland University Hospital (Homburg, Germany) and Norwegian Institute of Public Health (Oslo, Norway). We are grateful to Professor Sven Ove Samuelsen (Institute of Mathematics, University of Oslo and Division of Epidemiology, Norwegian Institute of Public Health) for valuable suggestions and recommendations on calculation procedures.

\section{References}

[1] V. Spiehler, Drugs in Saliva, in: A. C. Moffat, M. D. Osselton, and B. Widdop (Eds.), Clarke's Analysis of Drugs and Poisons, 3rd ed., Pharmaceutical Press, London, 2004, pp. 109-123.

[2] E.J. Cone, Legal, workplace, and treatment drug testing with alternate biological matrices on a global scale. Forensic Sci. Int. 121 (2001) 7-15.

[3] Y.H. Caplan, B.A. Goldberger, Alternative specimens for workplace drug testing. J. Anal. Toxicol. 25 (2001) 396-399.

[4] R.E. Choo, M.A. Huestis, Oral fluid as a diagnostic tool, Clin. Chem. Lab. Med. 42 (2004) 1273-1287.

[5] N. Samyn, A. Verstraete, C. van Haeren and P. Kintz, Analysis of drugs of abuse in saliva, Forensic Sci. Rev. 11 (1999) 2-17.

[6] E.L. Øiestad, U. Johansen and A.S. Christophersen, Drug screening of preserved oral fluid by liquid chromatography-tandem mass spectrometry, Clin. Chem. 53 (2007) 300-309. 
[7] K. Langel, C. Engblom, A. Pehrsson, T. Gunnar, K. Ariniemi and P. Lillsunde, Drug testing in oral fluid-evaluation of sample collection devices, J. Anal. Toxicol. 32 (2008) 393-401.

[8] D.J. Crouch, Oral fluid collection: the neglected variable in oral fluid testing, Forensic Sci. Int. 150 (2005) 165-173.

[9] S.M.R. Wille, E. Raes, P. Lillsunde, T. Gunnar, M. Laloup, N. Samyn, A.S. Christophersen, M.R. Moeller, K.P. Hammer and A. Verstraete, Relationship between oral fluid and blood concentrations of drugs of abuse in drivers suspected of DUID, Ther. Drug. Monit. 31(2009). 511-519

[10] H.G. Giles, R. Miller, S.M. Macleod and E.M. Sellers, Diazepam and Ndesmethyldiazepam in saliva of hospital inpatients, J. Clin. Pharmacol. 20 (1980) 7176.

[11] C. Engblom, T. Gunnar, A. Rantanen and P. Lillsunde, Driving under the influence of drugs - amphetamine concentrations in oral fluid and whole blood samples, J. Anal. Toxicol. 31 (2007) 276-280.

[12] J.G. Ramaekers, M.R. Moeller, P. van Ruitenbeek, E.L. Theunissen, E. Schneider and G. Kauert, Cognition and motor control as a function of Delta9-THC concentration in serum and oral fluid: limits of impairment, Drug Alcohol Depend. 85 (2006) 114-122.

[13] K. Kato, M. Hillsgrove, L. Weinhold, D.A. Gorelick, W.D. Darwin and E.J. Cone, Cocaine and metabolite excretion in saliva under stimulated and nonstimulated conditions, J. Anal. Toxicol. 17 (1993) 338-341.

[14] R.J. Schepers, J.M. Oyler, R.E. Joseph, Jr., E.J. Cone, E.T. Moolchan and M.A. Huestis, Methamphetamine and amphetamine pharmacokinetics in oral fluid and plasma after controlled oral methamphetamine administration to human volunteers, Clin. Chem. 49 (2003) 121-132.

[15] C.L. O'Neal, D.J. Crouch, D.E. Rollins and A.A. Fatah, The effects of collection methods on oral fluid codeine concentrations, J. Anal. Toxicol. 24 (2000) 536-542.

[16] A.G. Verstraete, E Raes. Rosita-2 project - Final report, in: Verstraete AG, Raes E, (Eds.) Academia Press, Ghent, 2006.

[17] H. Gjerde, P.T. Normann, B.S. Pettersen, T. Assum, M. Aldrin, U. Johansen, L. Kristoffersen, E.L. Øiestad, A.S. Christophersen and J. Mørland, Prevalence of alcohol and drugs among Norwegian motor vehicle drivers: A roadside survey, Accid. Anal. Prev. 40 (2008) 1765-1772. 
[18] N. Metropolis, S. Ulam, The Monte Carlo method, J. Amer. Stat. Assoc. 44 (1949) 335-341.

[19] J.G. Amar, The Monte Carlo method in science and engineering, Comp. Sci. Eng. 8 (2006) 9-19. 


\section{Figures}

Determine the meanlog $\mathrm{M}_{\mathrm{OF}}$ and $s d \log \mathrm{S}_{\mathrm{OF}}$ of drug concentrations in oral fluid of the study population.

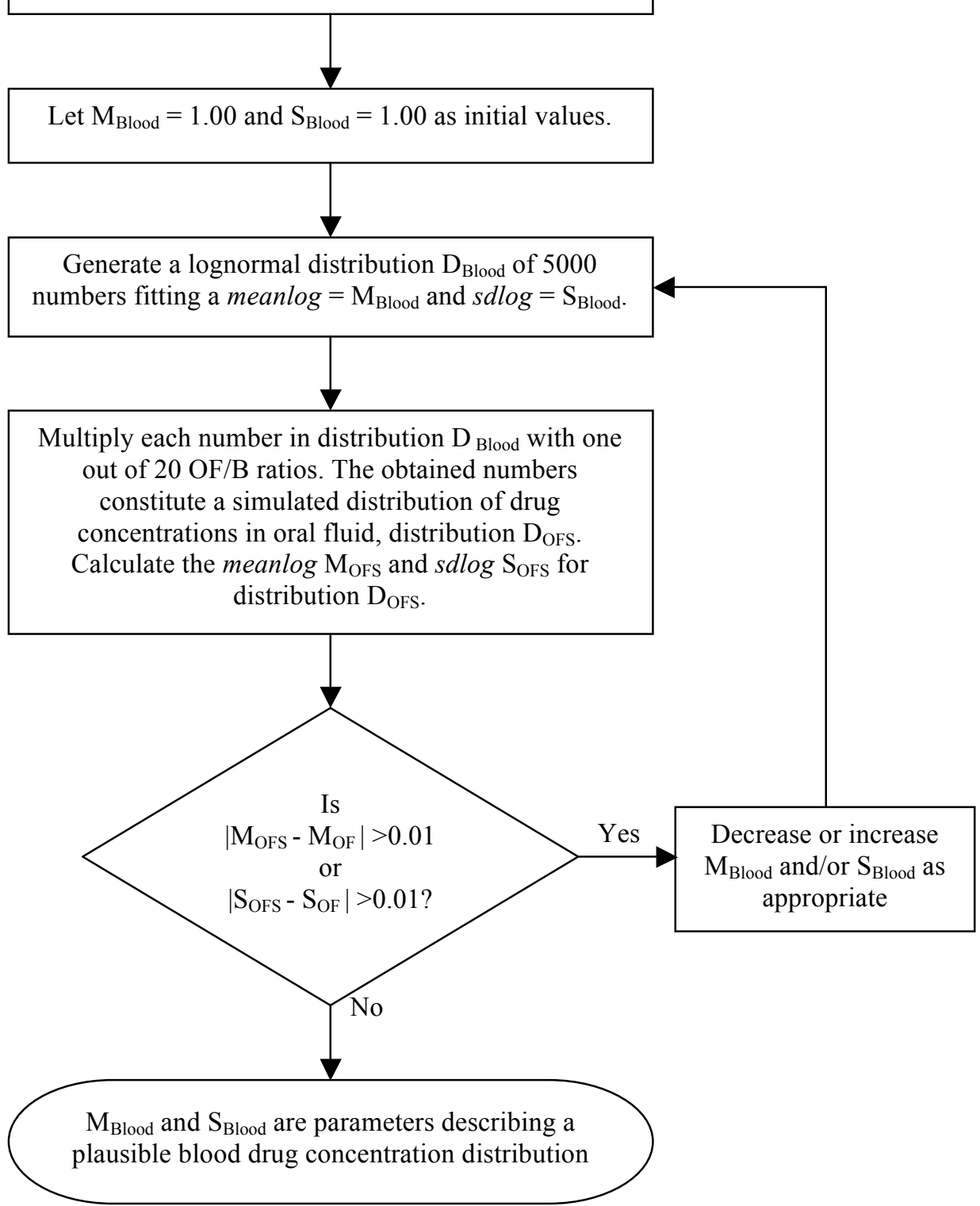

Figure 1. Flow diagram for the estimation of a plausible lognormal distribution of blood drug concentrations in a population based on drug concentrations in oral fluid. $\mathrm{M}_{\mathrm{OF}}$ and $\mathrm{S}_{\mathrm{OF}}$ are the meanlog and sdlog for the observed drug concentrations in oral fluid. $\mathrm{M}_{\mathrm{Blood}}$ and $\mathrm{S}_{\mathrm{Blood}}$ are the chosen meanlog and sdlog for drug concentrations in blood. $\mathrm{M}_{\mathrm{OFS}}$ and $\mathrm{S}_{\mathrm{OFS}}$ are the meanlog and $s d \log$ for the simulated drug concentration in oral fluid calculated by using drug concentrations in blood. 


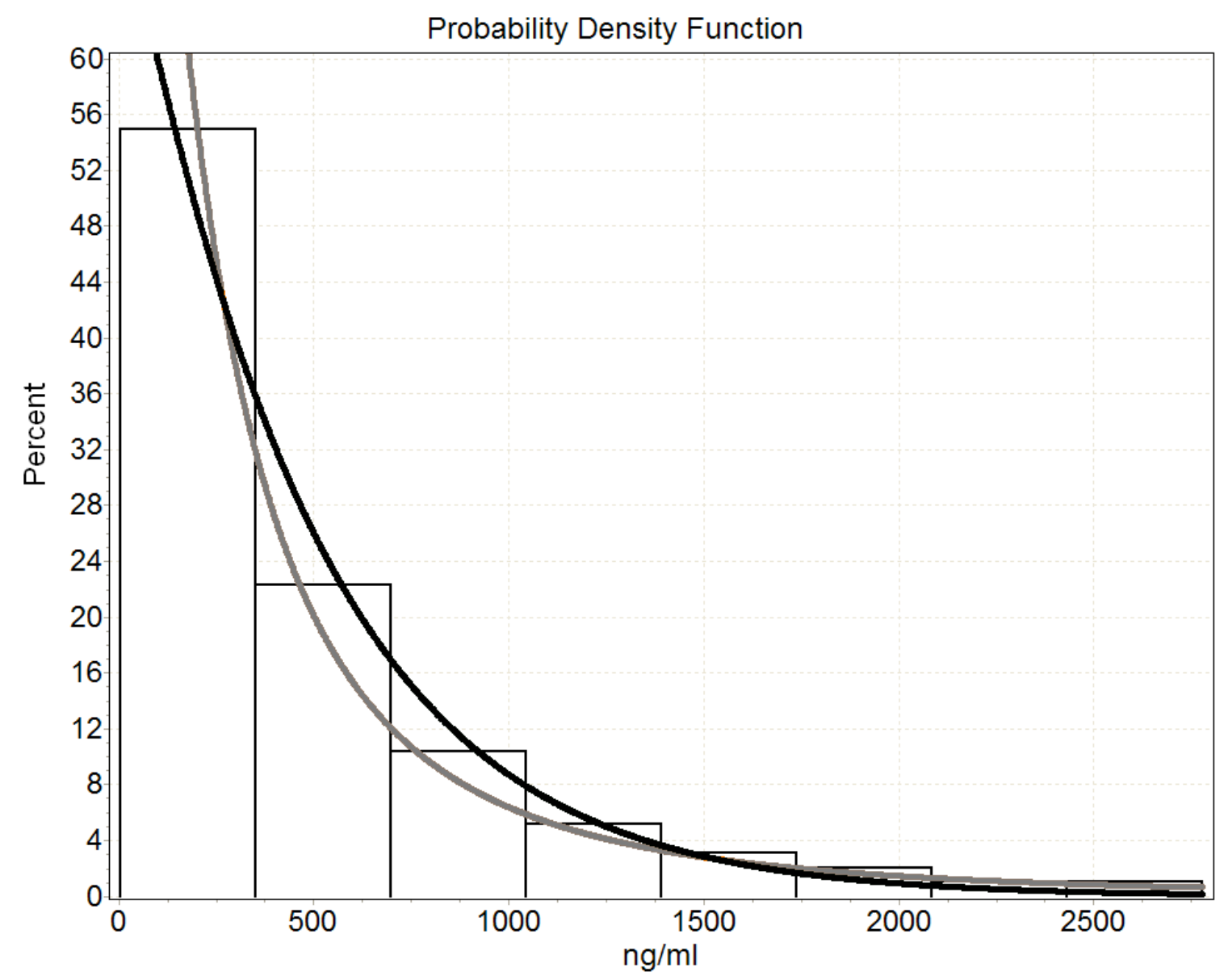

Amphetamine in blood

Lognormal W Weibull

Figure 2. Observed distributions of amphetamine concentrations in blood in Rosita-2 project and best fitted Lognormal and Weibull distributions. 